\title{
Mechanical properties change of thermoplastic elastomer after using of different dosage of irradiation by beta rays
}

\author{
Ales Mizera ${ }^{1, a}$, Miroslav Manas ${ }^{1}$, David Manas ${ }^{1}$, Pavel Stoklasek ${ }^{1}$, Martin Bednarik ${ }^{1}$ and Lenka Hylova ${ }^{1}$ \\ ${ }^{1}$ Tomas Bata University in Zlin, nam. T. G. Masaryka 5555, 76001 Zlin, Czech Republic
}

\begin{abstract}
Radiation processing of polymers is a well-established and economical commercial method of precisely modifying the properties of polymers. The industrial applications of the radiation processing of plastics and composites include polymerization, cross-linking, degradation and grafting. Radiation processing mainly involves the use of electron beams from electron accelerators. The Thermoplastic Elastomer (TPE) was used in this research and the mechanical properties were investigated at the ambient temperature. Results demonstrate that TPE has higher values of tensile strength with the increased irradiation dose and it has decreased elongation at break. This behaviour leads to the expansion of these materials in the automotive and electrical industry.
\end{abstract}

\section{Introduction}

Polymers rank among a construction materials which find use in industry branches. The advantage of polymers is a low weight together with the excellent mechanical properties, very good chemical resistance and other properties, which assign them for various applications. Disadvantage is mainly low temperature stability which significantly reduces usage of these polymers.

Every property improvement especially temperature stability helps to increase application possibilities. In addition, property modifications of standard polymers, which are relatively cheap products, give them advantage for another usage.

The cross-linking of rubbers and thermoplastic polymers is a well-proven process of the improvement of the thermal properties. The chemical cross-linking or rubber vulcanization is normally induced by the effect of heating after processing with the presence of a curing agent. The cross-linking process for thermosets is very similar. In thermosets the polymer molecules are also chemically linked due to heat after processing. Crosslinked rubbers have a wide-meshed molecular network that keeps them soft and their properties change only slightly on a wide temperature scale. On the other hand, thermosets are characterized by a very narrow-meshed network. Due to this fact they hardly change their high level of stiffness on a wide temperature scale [1].

Radiation cross-linking with accelerating electrons offers several advantages when compared to other radiation sources, particularly gamma rays and X-rays. The process is very fast, clean and can be controlled with a great deal of precision. In contrast to gamma rays and X-rays the electron beam can be steered relatively easy, thus allowing irradiation of variety of physical shapes.

\footnotetext{
${ }^{\mathrm{a}}$ Corresponding author: mizera@fai.utb.cz
}

The large advantage includes flexibility and controllability in operation, beneficial economics, high throughput capability and the ability to switch off the source of radiation $[1,2]$.

\section{„Upgrading" by Radiation Crosslinking}

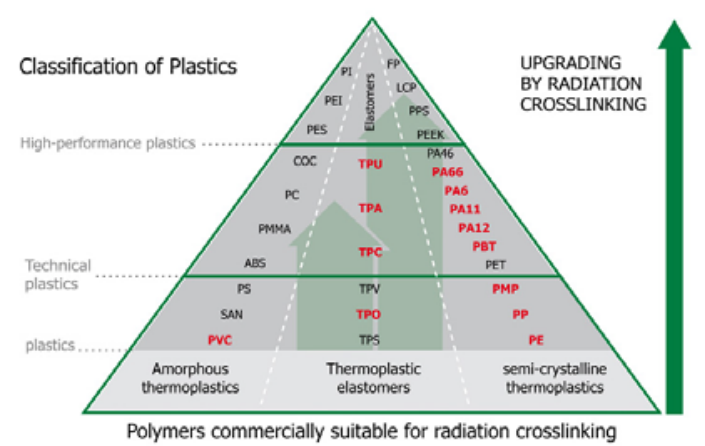

Figure 1. Pyramid of polymers [1].

The thermoplastics which are used for production of various types of products have very different properties. The special group of polymers which exhibit elastic behaviour to similar that of vulcanized conventional elastomer and can be processed as thermoplastics (i.e. by melt processing) are called thermoplastic elastomers (TPEs). The most thermoplastic elastomers are essentially phase-separated systems. Usually one phase is hard and solid at ambient temperature whereas the other is an elastomer. Often, the phases are bonded chemically by block or graft polymerization. In other case a fine dispersion of the phases is apparently sufficient. The hard phase gives these TPEs their strength and represents physical crosslinking. Without it the elastomer phase would be free to flow under stress and the polymer would be practically unusable. TPE has good physical properties 
of elastomers along processing characteristics of thermoplastic $[3,4]$.

A team of Indian scientists examined the influence of electron beam technology on blends of polyethylene and ethylene-vinyl acetate copolymer. These scientists discovered that tensile strength and E-modulus increased while elongation at the break initially increased followed by decrease with increase in the radiation dose at the constant monomer level and blend ratio [4]. LópezQuintana from Spain and his colleagues investigated the effect of beta radiation on mechanical properties of metallocene elastomers/PA 6 blends and they found out that TPE with excellent mechanical properties and thermal stability can be obtained by beta irradiation technology [5]. In Indian institute of technology was examined the effect of electron beam irradiation on silicafilled LDPE-EVA based thermoplastic elastomer. They discovered that all filled TPE system represented rise of E-modulus with increase in radiation dose and that rise of E-modulus with the increase in radiation dose is attributed to radiation cross-linking of the silica-filled TPE system [6].

This research paper deals with a possibility of using radiation cross-linked TPE as a suitable material for pipes in automotive industry, especially from point of view mechanical testing and temperature stability.

\section{Experimental}

As the basic polymer material was used thermoplastic elastomer (TPE, V-PTS-UNIFLEX-E25D/M*M800/20 natur). An ARBURG Allrounder 420C Advance Injection molding machine was used for sample preparation, with the processing conditional to comply with the TPE producer's recommendations.

Irradiation of tested TPE was performed with the kind help of BGS Germany, in the BGS Wiehl plant using accelerated electrons with a dosage range of 0 to $198 \mathrm{kGy}$. Samples with the dosage of irradiation higher than 99 $\mathrm{kGy}$ were exposed temperature $200^{\circ} \mathrm{C}$ during one hour and then these samples were cooled spontaneously to ambient temperature.

Mechanical properties before and after temperature load and temperature stability of irradiated thermoplastic elastomer after irradiation has been tested. The thermomechanical properties were measured. Perkin - Elmer Thermal Analyser TMA7 was used for the thermomechanical analysis, heated from $50^{\circ} \mathrm{C}$ to $400^{\circ} \mathrm{C}$ at $20^{\circ} \mathrm{C} / \mathrm{min}$, hold for $1 \mathrm{~min}$ at $50^{\circ} \mathrm{C}$. The tensile behaviour of temperature non-loaded and loaded samples of TPE were measured at the ambient temperature. Tensile test was carried out on tensile test machine ZWICK 1456 for TPE according to standard CSN EN ISO 527-1, 527-2 with used rate $50 \mathrm{~mm} / \mathrm{min}$. Test data was processed by Test Expert Standard software and tensile strength [MPa] and elongation at break [\%] were determined. The test of hardness was measured using a HPE - A Berreiss hardness tester type, Shore A method. The shape and dimensions of the testing samples were according to the CSN 621431 standards.

\section{Results and discussion}

\subsection{Temperature stability}

Temperature stability of polymers is very low in comparison with other construction materials. Experiments done in this project show that irradiation cross-linking markedly affects the temperature stability of studied TPE. The higher is the dose of irradiation the best is temperature stability of this polymers.

Irradiation affects the thermomechanical properties of the studied TPE (Figure 2). Already small dose of radiation (up to $33 \mathrm{kGy}$ ) move the softening point up to $210{ }^{\circ} \mathrm{C}$. TPE irradiated by the dose of $198 \mathrm{kGy}$ evinces the significant improvement of the temperature stability. Irradiation TPE with the dose $198 \mathrm{kGy}$ holds temperature stability up to $360{ }^{\circ} \mathrm{C}$ for short time. The temperature stability of TPE was measured. The temperature stability was evaluated by visual observation in the temperature chamber. The visual observation of sample behaviour after two hours exposition at $200{ }^{\circ} \mathrm{C}$ is given on Figure 3 .

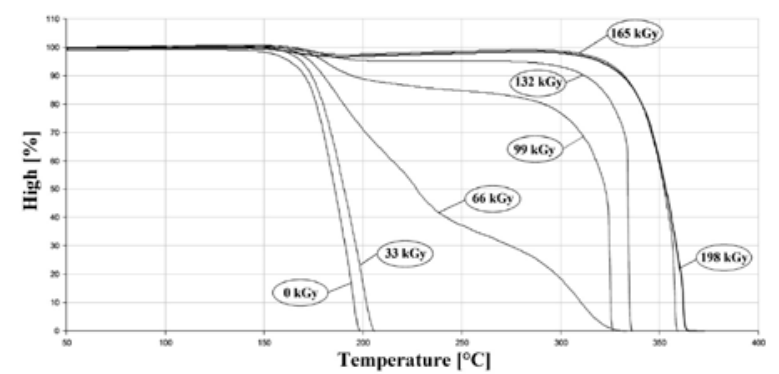

Figure 2. TPE thermomechanical analysis.

Specimens are fitted horizontally in the temperature chamber and loaded by the bending moment both form its own weight and the weight on the end of specimen. Specimen deformation is decreasing with increasing dose of radiation at elevated temperature (Figure 3). At $200{ }^{\circ} \mathrm{C}$ the specimen from not irradiated TPE is totally melted while the polymer irradiated by the dose of $198 \mathrm{kGy}$ keeps its cross-section without changes, there is only deformation by its own weight. The surface quality worsens.

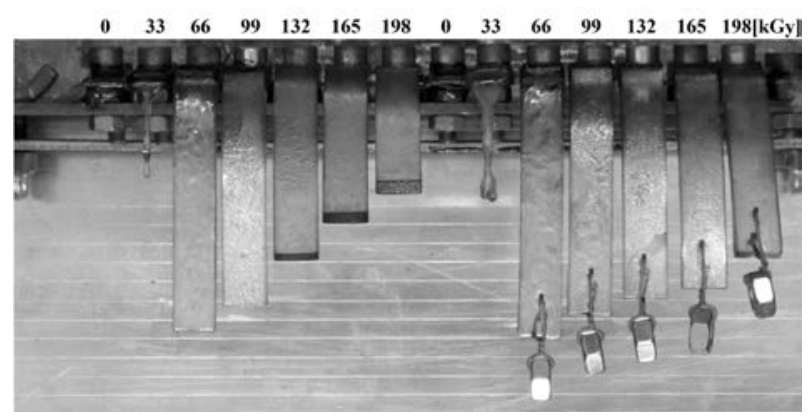

Figure 3. TPE specimen deformation after 1 hour temperature exposure $200^{\circ} \mathrm{C}$.

On Figure 4 is displayed surface of irradiated TPE by $99 \mathrm{kGy}$ after 15 minutes at $200{ }^{\circ} \mathrm{C}$. There is not any change of colour and surface quality. However, after 1 hour at the same temperature the surface changes colour 
to yellow-brown (Figure 5) and also surface quality is worse.

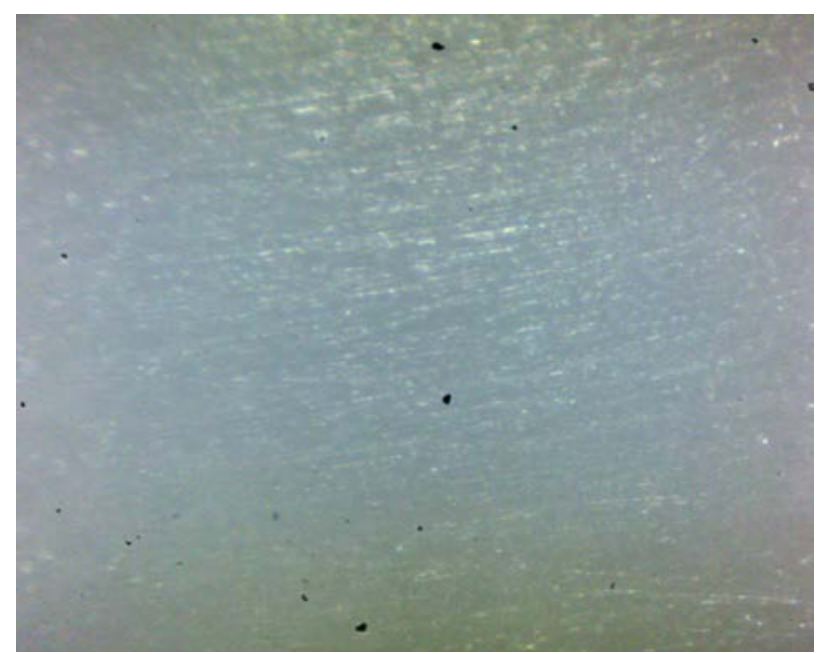

Figure 4. 100 times magnified surface of TPE irradiated by 99 kGy after 15 minutes at $200{ }^{\circ} \mathrm{C}$.

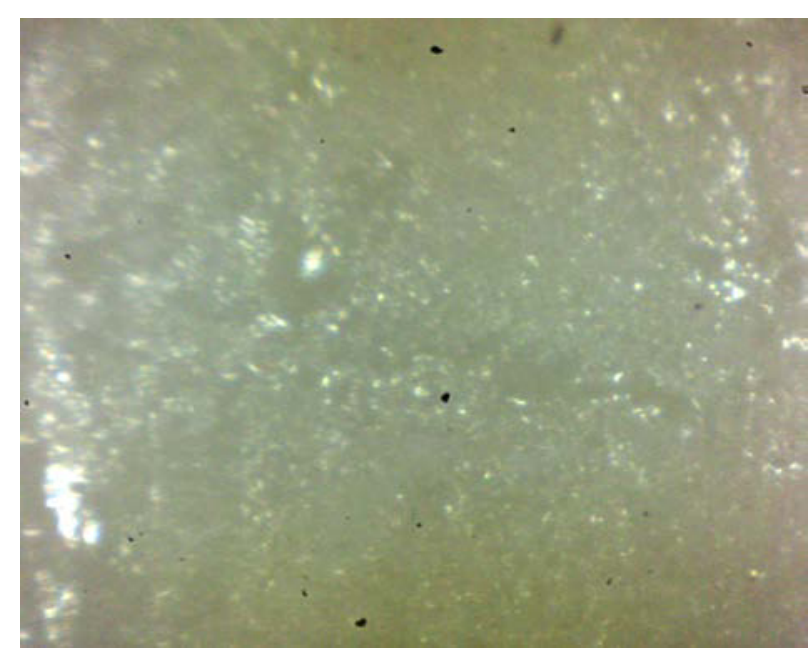

Figure 5. 100 times magnified surface of TPE irradiated by 99 kGy after 1 hour at $200{ }^{\circ} \mathrm{C}$.

On another pictures (Figure 6 and Figure 7) there are depicted similar changes of surface quality after 15 and 60 minutes. However, irradiated TPE with the dose 198 kGy after 1 hour at $200{ }^{\circ} \mathrm{C}$ has smoother surface than irradiated TPE by $99 \mathrm{kGy}$. It can be caused higher degree of cross-linking bonds.

\subsection{Tensile behaviour}

The tensile behaviour of TPE, before and after irradiation, was studied. For easier of evaluation of the measured data, and the comparison of the irradiated polymer with a non-irradiated one, the changes of measured were used on some graphs. The property of the non-irradiated polymer had the value of $100 \%$, while others were expressed in $\%$ as the ratio of measured property of irradiated polymers to the same property of non-irradiated polymer.

The tensile strength rises more than $40 \%$ after irradiation with the dose of $66 \mathrm{kGy}$ (Figure 9). Although the gel content (Figure 8) rises with the dose of irradiation the tensile strength goes down with the dose higher than $66 \mathrm{kGy}$. Then with the dose of $132 \mathrm{kGy}$ and higher the value of tensile strength oscillates around the value of not irradiated polymer. From the point of view of tensile strength the dose of irradiation of $66 \mathrm{kGy}$ looks to be optimal.

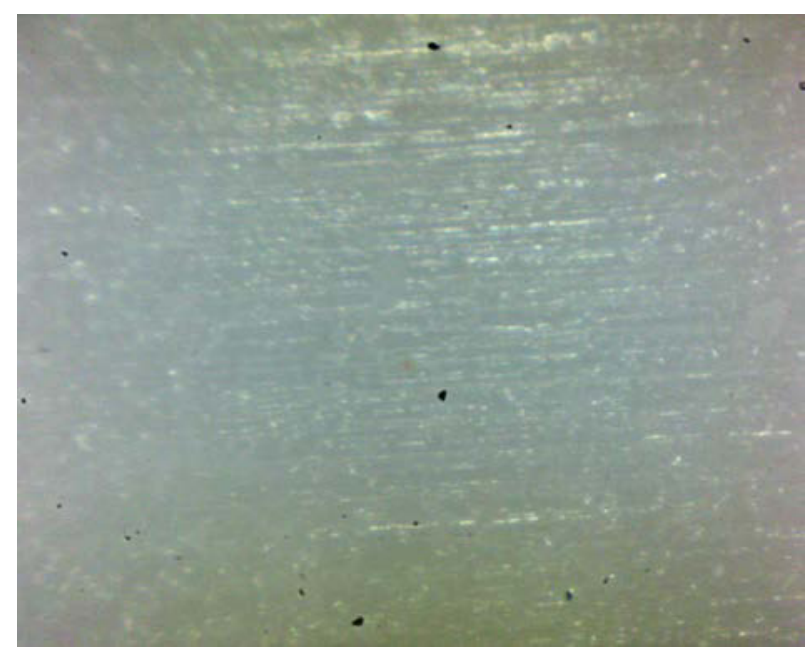

Figure 6. 100 times magnified surface of TPE irradiated by 198 kGy after 15 minutes at $200^{\circ} \mathrm{C}$.

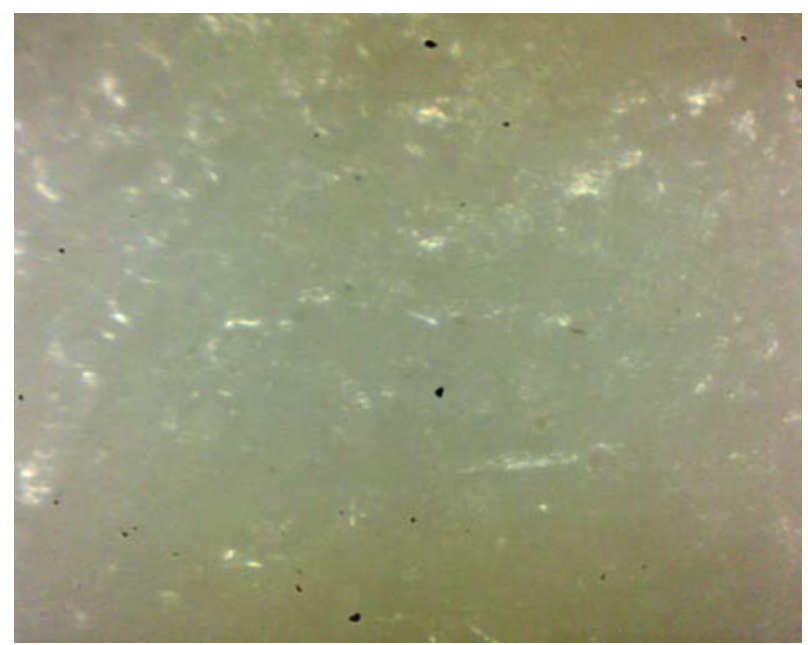

Figure 7. 100 times magnified surface of TPE irradiated by 198 kGy after 1 hour at $200^{\circ} \mathrm{C}$.

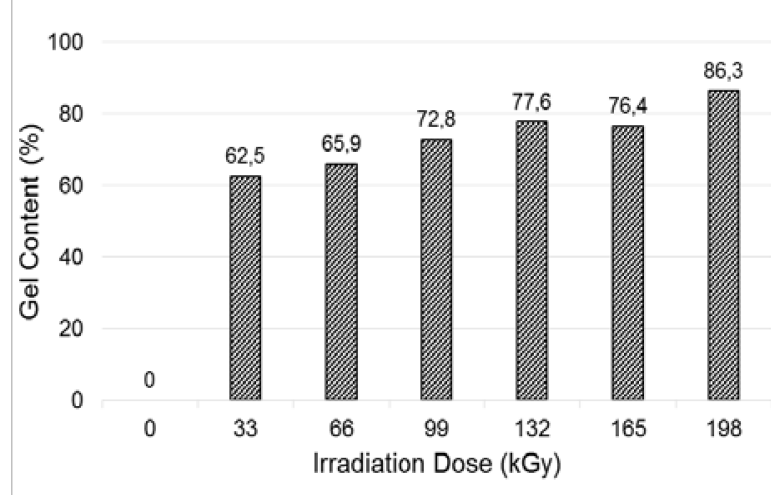

Figure 8. Gel content of irradiated TPE by beta rays.

Tensile strength of TPE after temperature load with increasing dose of irradiation decreases. However, the value of tensile strength is about $2 \mathrm{MPa}$ lover in comparison with temperature non-loaded TPE which was irradiated by $99 \mathrm{kGy}$. 
Elongation at break of TPE goes down continually with the dose of irradiation. By the highest applied dose of irradiation $(198 \mathrm{kGy})$ the elongation is only $22 \%$ of former (non-irradiated) polymer before temperature load. Due to irradiation of the studied TPE loses significantly its flexibility (Figure 10). After temperature load there is the same tendency as before temperature load.

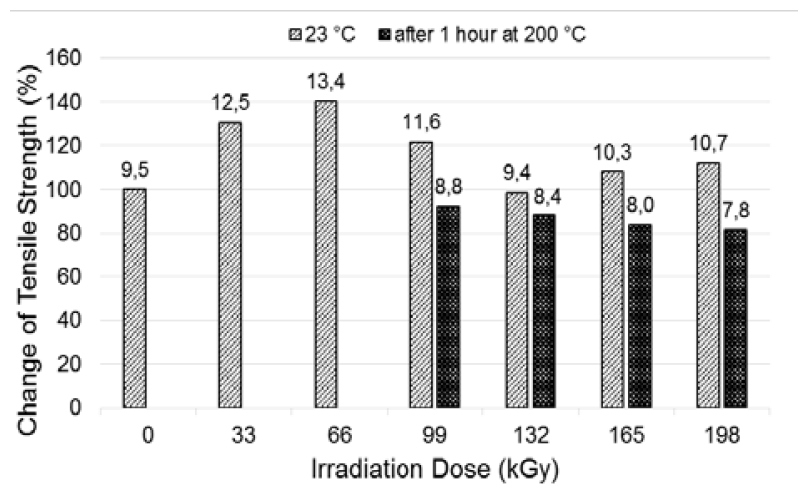

Figure 9. Change of tensile strength before and after temperature load.

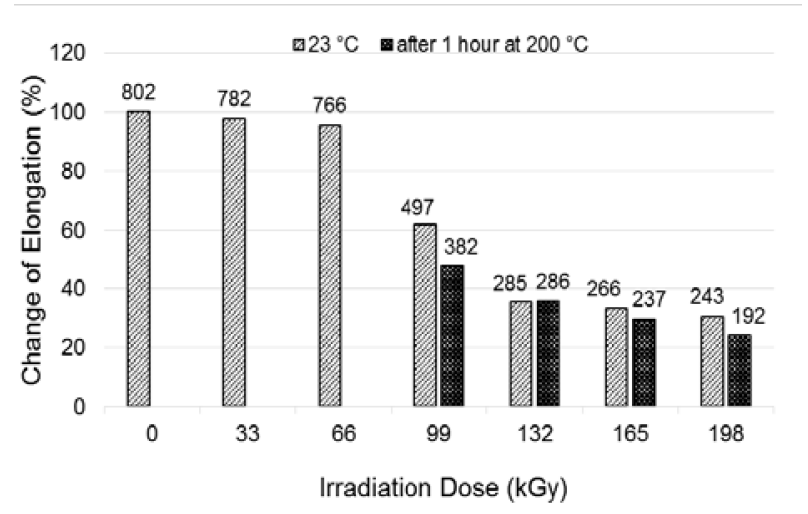

Figure 10. Change of elongation at break before and after temperature load.

\subsection{Hardness}

Change of Shore A hardness is showed on the Figure 11. Shore A hardness rises with increasing dose of irradiation up to $66 \mathrm{kGy}$ then decreases slowly along irradiation.

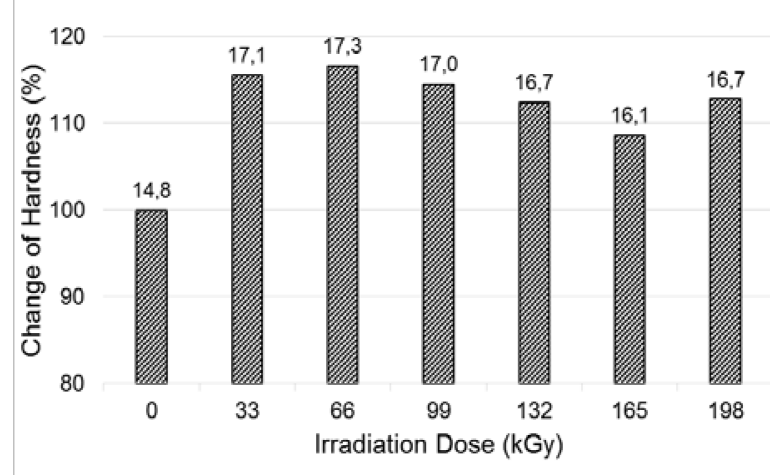

Figure 11. Change of Shore A hardness before and after temperature load.

\section{Summary}

Plastics are by far the largest group of polymeric materials being processed by electron beam irradiation. Cross-linking of polyolefins, PVC, polyesters, polyurethanes, polyamides, fluoropolymers and fiberreinforced composites are a common practice. Radiation cross-linking of polyamides requires considerably less overall energy and space, and is faster, more efficient and more environmentally acceptable. The disadvantage of electron beam cross-linking is a more or less nonuniform dose distribution. This can happen, particularly in thicker objects, due to intrinsic dose-depth profiles of electron beams. Another problem can be a nonuniformity of rotation of cylindrical objects as they traverse a scanned electron beam. However, the mechanical properties often depend on the mean cross-link density.

Results of the measurements of TPE after irradiation show significant changes of mechanical and thermomechanical properties. Tensile strength rises of 40 $\%$ after irradiation by the dose of $66 \mathrm{kGy}$. Elongation at break is going down gradually with the dose of irradiation. The lowest value of elongation at break was registered after irradiation by the dose of $198 \mathrm{kGy}$ and reaches only $25 \%$ of elongation of non-irradiated polymer. Very important is improvement of temperature stability of TPE after irradiation. Tested polymer irradiated by the dose of $198 \mathrm{kGy}$ shows no dimensional changes even by the temperature of $200{ }^{\circ} \mathrm{C}$. This significantly moves application possibilities of TPE to area with service temperature much higher than former melting point of tested polymers.

\section{Acknowledgment}

This paper is supported by the internal grant of TBU in Zlin No. IGA/FT/2016/010 funded from the resources of specific university research and by the Ministry of Education, Youth and Sports of the Czech Republic within the National Sustainability Programme project No. LO1303 (MSMT-7778/2014) and also by the European Regional Development Fund under the project CEBIATech No. CZ.1.05/2.1.00/03.0089.

\section{References}

1. J.G. Drobny, Radiation Technology for Polymers (CRC Press, Boca Raton, 2003)

2. D.W. Clegg, A.A. Collyer, Irradiation effects on polymers (Elsevier Applied Science, London, 1991)

3. J.G. Drobny, Handbook of Thermoplastic Elastomers (William Andrew Publishing, Norwich NY, 2007)

4. S. Chattopadhyay, T.K. Chaki, Anil K. Bhowmick, J. Appl. Polym. Sci. 79, 1877 (2001)

5. S. López-Quintana, C. Rosales, I. Gobernado-Mitre, J.C. Merino, J.M. Pastor, Polymer 45, 8041 (2004)

6. S. Hui, S. Mushtaq, T.K. Chaki, S. Chattopadhyay, J. Appl. Polym. Sci. 119, 8041 (2004) 